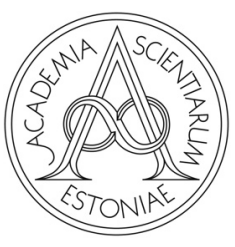

Proceedings of the Estonian Academy of Sciences,

$2022,71,1,65-76$

https://doi.org/10.3176/proc.2022.1.06

Available online at www.eap.ee/proceedings

INTERVAL

COMPUTATIONS

\title{
Gershgorin disk theorem in complex interval matrices
}

\author{
This article is dedicated to the 100th birthday of Ülo Lepik
}

Suman Maiti and Snehashish Chakraverty*

Department of Mathematics, National Institute of Technology Rourkela, Odisha - 769008, India

Received 8 July 2021, accepted 28 September 2021, available online 9 February 2022

(C) 2022 Authors. This is an Open Access article distributed under the terms and conditions of the Creative Commons Attribution 4.0 International License CC BY 4.0 (http://creativecommons.org/licenses/by/4.0).

\begin{abstract}
In this article, the Gershgorin disk theorem in complex interval matrices is proposed for enclosing interval eigenvalues. This is a non-iterative method for finding eigenvalue bounds for both real and imaginary parts. Moreover, we are able to find gaps between the clusters of interval eigenvalues and have compared the results with the previous theorems for interval eigenvalue bounds for complex interval matrices. These results can be decisive for checking Hurwitz and Schur stability of complex interval matrices that appear in uncertain dynamical systems. Further bounds obtained from the present formulae can be considered as the initial bounds for iterative methods.
\end{abstract}

Key words: complex interval matrices, Gershgorin disk theorem, interval eigenvalues, eigenvalue bounds.

\section{INTRODUCTION}

Finding eigenvalues of matrices is a fundamental problem in linear algebra, and it concerns numerous issues of engineering computations. In practical situations, the bounded uncertain error appears and influences us to calculate interval eigenvalues of interval matrices. Computing exact eigenvalue bounds for interval matrices is a challenging task. Therefore, we compute outer eigenvalue bounds.

Checking the stability of interval matrices is an NP-hard problem. To determine the stability of the uncertain dynamical systems, we need to compute tighter interval eigenvalue bounds of interval matrices that appear in uncertain dynamical systems [1]. Hurwitz and Schur stability of interval matrices, studied in [1,2], can be determined by computing the eigenvalues of vertex matrices. Stability analysis of a fractional-order linear time-invariant uncertain system can be performed by calculating eigenvalues of Hermitian complex interval matrix as shown by Ahn et al. [3]. Other applications of computing tighter eigenvalue bounds of interval matrices correlated to the problems with bounded uncertainty are a spring-mass system [4,5], a nine-bar truss [4], etc.

As regards the theorems proposed by Deif [6] for exact eigenvalues of both symmetric and general interval matrices with the condition of sign invariance of eigenvectors computed at the centre matrix, it is difficult to ensure the sign invariance of the eigenvectors of the centre matrix, and this restricts the applicability of the theorems. Hertz [1] exactly enclosed the interval eigenvalues of symmetric interval matrices by

\footnotetext{
*Corresponding author, sne_chak@yahoo.com
} 
calculating the eigenvalues of vertex matrices, which is an exponential method. Rohn [7] further proposed a theorem for a cheap bound of symmetric interval matrices. Based on Weyl's and Gershgorin's theorems, Leng and $\mathrm{He}$ [4] gave accessible eigenvalue bounds for symmetric interval matrices. One drawback of these two methods is that the width of each eigenvalue bound is the same. In addition, Hladík et al. [8] provided eigenvalue bounds of symmetric interval matrices by using Cauchy's interlacing property.

The bounds for both real and imaginary parts of eigenvalues of general interval matrices can be obtained by the Rohn formulae [9], although it is overestimated. Continuity property of characteristic polynomial allows us to calculate tighter eigenvalue bounds for interval matrices [5]. Hladík et al. [8] produced bounds for the real part of all eigenvalues of interval matrices by manipulating the Bauer-Fike theorem [10,11]. They also proposed [12] a filtering algorithm that can give a tight real eigenvalue bound. Mayer [13] presented the enclosure for eigenvalues of real and complex interval matrices from Taylor expansion. The eigenvalue bounds for complex interval matrices are presented in [14]. Hladík [15] gave formulae for bounding both real and imaginary parts of eigenvalues of complex interval matrices, which need to compute eigenvalue bounds of a double dimensional symmetric interval matrix. The tightness of the bound depends on the solver for the symmetric interval matrix. Two different approaches for bounding eigenvalues of complex interval matrices are presented in [16]. Sufficient conditions for regularity of complex interval matrices were derived, and using those conditions, iterative processes were developed for bounding eigenvalues [17].

Our goal is to compute tighter enclosures for eigenvalues of complex interval matrices. Gershgorin disks of small radius become helpful for tighter enclosures of eigenvalues of complex interval matrices. Moreover, disjoint Gershgorin disks can separate the eigenvalue clusters of complex interval matrices. We have proposed theorems based on the Gershgorin disk theorem that can bound separately real and imaginary parts of eigenvalues of complex interval matrices. Also, these bounds may be able to find the gaps between the eigenvalue clusters.

In Section 2, we recall the preliminaries of interval computations. Section 3 introduces different approaches for getting eigenvalue bounds of symmetric, non-symmetric, and complex interval matrices. In Section 4 we derive eigenvalue bounds for complex interval matrices. In Section 5, different examples are presented and their eigenvalue bounds are compared. In Section 6 an application is demonstrated.

\section{PRELIMINARIES}

An interval is denoted by $\boldsymbol{a}$ and defined by a closed interval $[\underline{a}, \bar{a}]$ in $\mathbb{R}$. The arithmetic of two intervals $\boldsymbol{a}=[\underline{a}, \bar{a}]$ and $\boldsymbol{b}=[\underline{b}, \bar{b}]$ is defined as follows:

$\boldsymbol{~} \boldsymbol{a}+\boldsymbol{b}=[\underline{a}+\underline{b}, \bar{a}+\bar{b}]$,

$-\boldsymbol{a}=[-\bar{a},-\underline{a}]$,

- $\boldsymbol{a} . \boldsymbol{b}=[\min (\underline{a b}, \underline{a b}, \bar{a} \underline{b}, \bar{a} \bar{b}), \max (\underline{a b}, \underline{a} \bar{b}, \bar{a} \underline{b}, \bar{a} \bar{b})]$,

- $1 / \boldsymbol{a}=[1 / \bar{a}, 1 / \underline{a}]$, provided $0 \notin \boldsymbol{a}$.

The centre, radius, and absolute of an interval $\boldsymbol{a}$ are denoted by $a_{c}, a_{\triangle}$, and $|\boldsymbol{a}|$ and defined by $(\underline{a}+\bar{a}) / 2$, $(\bar{a}-\underline{a}) / 2$, and $\max \{|\underline{a}|,|\bar{a}|\}$, respectively [18].

An interval matrix is defined as $\boldsymbol{A}=\{A: \underline{A} \leq A \leq \bar{A}\}=\left(\boldsymbol{a}_{j k}\right)$, where $\underline{A}$ and $\bar{A}$ are the lower and upper matrices and inequalities are taken componentwise. The centre matrix, radius matrix, and absolute matrices of an interval matrix are denoted by $A_{c}, A_{\triangle}$, and $|\boldsymbol{A}|$ and defined by $(\underline{A}+\bar{A}) / 2,(\bar{A}-\underline{A}) / 2$, and $\left(\left|\boldsymbol{a}_{j k}\right|\right)$, respectively. $\boldsymbol{A}^{s}=\left\{A \in \boldsymbol{A} \mid A=A^{T}\right\}$ is the symmetric interval matrix corresponding to an interval matrix $\boldsymbol{A}$. The matrices $A=\left\{\left(a_{j k}\right): a_{j k}=a_{j k}\right.$ or $\left.\overline{a_{j k}}\right\}$ in this form are called vertex matrices in $\boldsymbol{A}$. We denote the set of all vertex matrices in $\boldsymbol{A}$ by $V \overline{(\boldsymbol{A})}$.

For real interval matrices $\boldsymbol{A}$ and $\boldsymbol{B}$ we can define complex interval matrix as $\boldsymbol{A}+i \boldsymbol{B}=\{A+i B: A \in \boldsymbol{A}$ and $B \in \boldsymbol{B}\}$. For a complex interval matrix, the eigenvalue set $\Lambda=\{\lambda+i \mu \in \mathbb{C}:(A+i B) z=(\lambda+i \mu) z$ and $z \in\left(\mathbb{C}^{n}\right)^{*}, A \in \boldsymbol{A}$ and $\left.B \in \boldsymbol{B}\right\}$. The centre, radius, and absolute matrices of the complex interval matrices $\boldsymbol{A}+i \boldsymbol{B}$ are defined as $A_{c}+i B_{c}, A_{\Delta}+B_{\Delta},|\boldsymbol{A}|+|\boldsymbol{B}|$. 


\section{COMPLEX INTERVAL EIGENVALUE PROBLEM}

Computing the exact eigenvalue set $\Lambda$ is a challenging task. In this context we can find outer bounds of the set $\Lambda$ corresponding to the complex interval matrix $\boldsymbol{A}+i \boldsymbol{B}$.

Hladík [15] used the following eigenvalue bounds of symmetric interval matrices given by Rohn and Hertz for enclosing eigenvalues of complex interval matrices. In the symmetric eigenvalue problem, all the eigenvalues are real, so the imaginary part of the eigenvalues is zero.

Theorem 1. [7] Let $\boldsymbol{A}^{s}=\left\{A \in \boldsymbol{A} \mid A=A^{T}\right\}$ be a symmetric interval matrix corresponding to an interval matrix $A$. Then

$$
\lambda_{i}\left(\boldsymbol{A}^{s}\right) \subseteq\left[\lambda_{i}\left(A_{c}\right)-\rho\left(A_{\triangle}\right), \lambda_{i}\left(A_{c}\right)+\rho\left(A_{\triangle}\right)\right],
$$

for $i=1, \ldots, n$.

Exact eigenvalue bounds for symmetric interval matrices can be obtained by calculating the eigenvalues of vertex matrices. The computational cost of this method is exponential.

Let $B^{n}=\left\{x \in \mathbb{R}^{n}:\|x\|=1\right\} . B_{i}=B^{n} \cap O_{i}$ for $i=1, \ldots, 2^{n-1}$, where $O_{i}$ are the orthants of $\mathbb{R}^{n}$. Here we need to consider $2^{n-1}$ orthants which are symmetric about the origin.

By the Rayleigh quotient we have

$$
\bar{\lambda}\left(\boldsymbol{A}^{s}\right)=\max _{A \in \boldsymbol{A}^{s}}\left(\max _{\|x\|=1}\left(x^{T} A x\right)\right)
$$

which can be easily computed by using the following theorem.

Theorem 2. $[1] \bar{\lambda}\left(\boldsymbol{A}^{\boldsymbol{s}}\right)=\max _{1 \leq i \leq 2^{n-1}} \bar{\lambda}^{i}$, where $\bar{\lambda}^{i}=\max _{x \in B^{n}} x^{T} \bar{A}^{i} x, \bar{A}^{i}=\left[\bar{a}_{k l}^{i}\right] \in V(\boldsymbol{A})$,

$$
\bar{a}_{k l}^{i}= \begin{cases}\bar{a}_{k k} & \text { if } k=l, \\ \bar{a}_{k l} & \text { if } x_{k} x_{l} \geq 0 \wedge k \neq l, \\ \underline{a}_{k l} & \text { if } x_{k} x_{l}<0 \wedge k \neq l,\end{cases}
$$

for $i=1, \ldots, 2^{n-1}$.

Similarly,

$$
\underline{\lambda}\left(\boldsymbol{A}^{\boldsymbol{s}}\right)=\min _{A \in \boldsymbol{A}^{s}}\left(\min _{\|x\|=1}\left(x^{T} A x\right)\right)
$$

and it can be easily computed by using the following theorem.

Theorem 3. [1] $\underline{\lambda}\left(\boldsymbol{A}^{s}\right)=\min _{1 \leq i \leq 2^{n-1}} \underline{\lambda}^{i}$, where $\underline{\lambda}^{i}=\min _{x \in B^{n}} x^{T} \underline{A}^{i} x, \underline{A}^{i}=\left[\underline{a}_{k l}^{i}\right] \in V(\boldsymbol{A})$,

$$
\underline{a}_{k l}^{i}= \begin{cases}\underline{a}_{k k} & \text { if } k=l, \\ \underline{a}_{k l} & \text { if } x_{k} x_{l} \geq 0 \wedge k \neq l, \\ \bar{a}_{k l} & \text { if } x_{k} x_{l}<0 \wedge k \neq l,\end{cases}
$$

for $i=1, \ldots, 2^{n-1}$.

Other easily computable eigenvalue bounds for symmetric interval matrices can be obtained from the following theorem. 
Theorem 4. [4] Let $\boldsymbol{A}^{s}=\left(\left[a_{i j}, \overline{a_{i j}}\right]\right)$ be an $n \times n$ real symmetric interval matrix. For $A \in \boldsymbol{A}^{s}$, the eigenvalue of $A x=\lambda x$ is ordered as $\lambda_{n} \overline{\leq} \cdots \leq \lambda_{1}$ and $A_{i j}=\max _{j \neq i}\left\{\left|\underline{a_{i j}}\right|,\left|\overline{a_{i j}}\right|\right\}$ for $i, j=1, \ldots, n$. Then

$$
\begin{gathered}
\lambda_{1} \leq \max _{i=1, \ldots, n}\left(\sum_{j \neq i} A_{i j}+A_{i i}\right), \\
-\max _{i=1, \ldots, n}\left(\sum_{j \neq i} A_{i j}+A_{i i}\right) \leq \lambda_{n} .
\end{gathered}
$$

In the following theorem, Rohn enclosed complex eigenvalues of a real interval matrix by computing eigenvalues of the matrices formed by the interval matrix's centre and radius matrices.

Theorem 5. [9] Let $\boldsymbol{A}=[\underline{A}, \bar{A}]$ be an $n \times n$ interval matrix. Consider $A z=(\lambda+i \mu) z$, where $A \in \boldsymbol{A}$ and $z$ $\in\left(\mathbb{C}^{n}\right)^{*}$. Then

$$
\begin{aligned}
& \lambda_{\min }\left(A_{c}\right)-\rho\left(A_{\triangle}\right) \leq \lambda \leq \lambda_{\max }\left(A_{c}^{\prime}\right)+\rho\left(A_{\triangle}^{\prime}\right), \\
& \lambda_{\min }\left(A_{c}^{\prime \prime}\right)-\rho\left(A_{\triangle}^{\prime \prime}\right) \leq \mu \leq \lambda_{\max }\left(A_{c}^{\prime \prime}\right)+\rho\left(A_{\triangle}^{\prime \prime}\right),
\end{aligned}
$$

where

$$
\begin{aligned}
A_{c} & =\frac{1}{2}(\underline{A}+\bar{A}) \text { and } A_{\triangle}=\frac{1}{2}(\bar{A}-\underline{A}), \\
A_{c}^{\prime} & =\frac{1}{2}\left(A_{c}+A_{c}^{T}\right) \text { and } A_{\triangle}^{\prime}=\frac{1}{2}\left(A_{\triangle}+A_{\triangle}^{T}\right), \\
A_{c}^{\prime \prime} & =\left(\begin{array}{cc}
0 & \frac{1}{2}\left(A_{c}-A_{c}^{T}\right) \\
\frac{1}{2}\left(A_{c}^{T}-A_{c}\right) & 0
\end{array}\right), \\
A_{\triangle}^{\prime \prime} & =\left(\begin{array}{cc}
0 & A_{\triangle}^{\prime} \\
A_{\triangle}^{\prime} & 0
\end{array}\right) .
\end{aligned}
$$

Hertz bounded complex eigenvalues of complex interval matrices with the following formulae of the below theorem.

Theorem 6. [14] Let $\boldsymbol{A}+i \boldsymbol{B}$ be a complex interval matrix. Then for each eigenvalue $\lambda+i \mu$, we have

$$
\begin{aligned}
& \lambda \leq \lambda_{1}\left(\frac{1}{2}\left(A_{c}+A_{c}^{T}\right)\right)+\rho\left(\frac{1}{2}\left(A_{\triangle}+A_{\triangle}^{T}\right)\right)+\lambda_{1}\left(\begin{array}{cc}
0 & \frac{1}{2}\left(B_{c}^{T}-B_{c}\right) \\
\frac{1}{2}\left(B_{c}-B_{c}^{T}\right) & 0
\end{array}\right)+\rho\left(\left(\begin{array}{cc}
0 & \frac{1}{2}\left(B_{\triangle}^{T}+B_{\triangle}\right) \\
\frac{1}{2}\left(B_{\triangle}+B_{\triangle}^{T}\right) & 0
\end{array}\right)\right), \\
& \lambda \geq \lambda_{n}\left(\frac{1}{2}\left(A_{c}+A_{c}^{T}\right)\right)-\rho\left(\frac{1}{2}\left(A_{\triangle}+A_{\triangle}^{T}\right)\right)+\lambda_{n}\left(\begin{array}{cc}
0 & \frac{1}{2}\left(B_{c}^{T}-B_{c}\right) \\
\frac{1}{2}\left(B_{c}-B_{c}^{T}\right) & 0
\end{array}\right)-\rho\left(\left(\begin{array}{cc}
0 & \frac{1}{2}\left(B_{\triangle}^{T}+B_{\triangle}\right) \\
\frac{1}{2}\left(B_{\triangle}+B_{\triangle}^{T}\right) & 0
\end{array}\right)\right),
\end{aligned}
$$

and

$$
\begin{aligned}
& \mu \leq \lambda_{1}\left(\frac{1}{2}\left(B_{c}+B_{c}^{T}\right)\right)+\rho\left(\frac{1}{2}\left(B_{\triangle}+B_{\triangle}^{T}\right)\right)+\lambda_{1}\left(\begin{array}{cc}
0 & \frac{1}{2}\left(A_{c}-A_{c}^{T}\right) \\
\frac{1}{2}\left(A_{c}^{T}-A_{c}\right) & 0
\end{array}\right)+\rho\left(\left(\begin{array}{cc}
0 & \frac{1}{2}\left(A_{\triangle}+A_{\triangle}^{T}\right) \\
\frac{1}{2}\left(A_{\triangle}^{T}+A_{\triangle}\right) & 0
\end{array}\right),\right. \\
& \left.\mu \geq \lambda_{n}\left(\frac{1}{2}\left(B_{c}+B_{c}^{T}\right)\right)-\rho\left(\frac{1}{2}\left(B_{\triangle}+B_{\triangle}^{T}\right)\right)+\lambda_{n}\left(\begin{array}{cc}
0 & \frac{1}{2}\left(A_{c}-A_{c}^{T}\right) \\
\frac{1}{2}\left(A_{c}^{T}-A_{c}\right) & 0
\end{array}\right)-\rho\left(\begin{array}{cc}
0 & \frac{1}{2}\left(A_{\triangle}+A_{\triangle}^{T}\right) \\
\frac{1}{2}\left(A_{\triangle}^{T}+A_{\triangle}\right) & 0
\end{array}\right)\right) .
\end{aligned}
$$

Using the Courant-Fischer theorem [19,20], Hladík proposed the following theorem to enclose the complex eigenvalues of a complex interval matrix. The efficiency of this theorem depends on the solver for the symmetric interval matrices given by different authors.

Theorem 7. [15] Let $\boldsymbol{A}, \boldsymbol{B} \in \mathbb{\mathbb { R } ^ { n \times n }}$. Then for every eigenvalue $\lambda+i \mu \in \Lambda(\boldsymbol{A}+i \boldsymbol{B})$, we have

$$
\begin{aligned}
& \underline{\boldsymbol{\lambda}}_{n}\left(\begin{array}{cc}
\frac{1}{2}\left(\boldsymbol{A}+\boldsymbol{A}^{T}\right) & \frac{1}{2}\left(\boldsymbol{B}^{T}-\boldsymbol{B}\right) \\
\frac{1}{2}\left(\boldsymbol{B}-\boldsymbol{B}^{T}\right) & \frac{1}{2}\left(\boldsymbol{A}+\boldsymbol{A}^{T}\right)
\end{array}\right)^{s} \leq \lambda \leq \bar{\lambda}_{1}\left(\begin{array}{cc}
\frac{1}{2}\left(\boldsymbol{A}+\boldsymbol{A}^{T}\right) & \frac{1}{2}\left(\boldsymbol{B}^{T}-\boldsymbol{B}\right) \\
\frac{1}{2}\left(\boldsymbol{B}-\boldsymbol{B}^{T}\right) & \frac{1}{2}\left(\boldsymbol{A}+\boldsymbol{A}^{T}\right)
\end{array}\right)^{s}, \\
& \underline{\boldsymbol{\lambda}}_{n}\left(\begin{array}{cc}
\frac{1}{2}\left(\boldsymbol{B}+\boldsymbol{B}^{T}\right) & \frac{1}{2}\left(\boldsymbol{A}-\boldsymbol{A}^{T}\right) \\
\frac{1}{2}\left(\boldsymbol{A}^{T}-\boldsymbol{A}\right) & \frac{1}{2}\left(\boldsymbol{B}+\boldsymbol{B}^{T}\right)
\end{array}\right)^{s} \leq \mu \leq \bar{\lambda}_{1}\left(\begin{array}{cc}
\frac{1}{2}\left(\boldsymbol{B}+\boldsymbol{B}^{T}\right) & \frac{1}{2}\left(\boldsymbol{A}-\boldsymbol{A}^{T}\right) \\
\frac{1}{2}\left(\boldsymbol{A}^{T}-\boldsymbol{A}\right) & \frac{1}{2}\left(\boldsymbol{B}+\boldsymbol{B}^{T}\right)
\end{array}\right)^{s} .
\end{aligned}
$$


There are few iterative methods [16,17] for computing eigenvalue bounds of complex interval matrices. The computational time of these algorithms depends on the initial bounds of the eigenvalues. Here we propose theorems for eigenvalue bounds of complex interval matrices which can be used as initial bounds for iterative methods.

Let $A=\left(a_{j k}\right)$ be an $n \times n$ complex matrix. For $j \in\{1, \ldots, n\}$, let $R_{j}=\sum_{k \neq j}\left|a_{j k}\right|$ be the sum of the absolute values of the non-diagonal entries in the $j^{t h}$ row. Each $D\left(a_{j j}, R_{j}\right) \subseteq \mathbb{C}$ is a closed disk centred at $a_{j j}$ with radius $R_{j}$ called a Gershgorin disk.

Theorem 8 (Gershgorin Disk Theorem). [21] Every eigenvalue of a matrix $A=\left(a_{j k}\right)$ lies within at least one of the Gershgorin disks $D\left(a_{j j}, R_{j}\right)$.

Now, we have extended the Gershgorin disk theorem to complex interval matrices for enclosing the eigenvalue set, and consequently derived Theorem 9 and Theorem 10. Their advantages can be seen by the examples provided in the next section.

\section{GERSHGORIN DISK THEOREM IN COMPLEX INTERVAL MATRICES}

Theorem 9. Let $\boldsymbol{A}+i \boldsymbol{B}$ be a complex interval matrix, where $\boldsymbol{A}=[\underline{A}, \bar{A}]$ and $\boldsymbol{B}=[\underline{B}, \bar{B}]$ are two real interval matrices. Then for every eigenvalue $\lambda+i \mu$ of $\boldsymbol{A}+i \boldsymbol{B}$, we have

$$
\begin{aligned}
& \underline{a_{j j}}-\sum_{k \neq j} \sqrt{\left|\left[\underline{a_{j k}}, \overline{a_{j k}}\right]\right|^{2}+\left|\left[\underline{b_{j k}}, \overline{b_{j k}}\right]\right|^{2}} \leq \lambda \leq \overline{a_{j j}}+\sum_{k \neq j} \sqrt{\left|\left[\underline{a_{j k}}, \overline{a_{j k}}\right]\right|^{2}+\left|\left[\underline{b_{j k}}, \overline{b_{j k}}\right]\right|^{2}}, \\
& \underline{b_{j j}}-\sum_{k \neq j} \sqrt{\mid\left[\left.\underline{\left.a_{j k}, \overline{a_{j k}}\right]}\right|^{2}+\mid\left[\left.\underline{\left.b_{j k}, \overline{b_{j k}}\right]}\right|^{2}\right.\right.} \leq \mu \leq \overline{b_{j j}}+\sum_{k \neq j} \sqrt{\mid\left[\left.\underline{\left.a_{j k}, \overline{a_{j k}}\right]}\right|^{2}+\left|\left[\underline{b_{j k}}, \overline{b_{j k}}\right]\right|^{2}\right.},
\end{aligned}
$$

for $j \in\{1, \ldots, n\}$.

Proof. For $j \in\{1, \ldots, n\}$ and $A+i B \in \boldsymbol{A}+i \boldsymbol{B}$, from Theorem 8 we have

$$
\begin{aligned}
& \left|(\lambda+i \mu)-\left(a_{j j}+i b_{j j}\right)\right| \leq \sum_{k \neq j} \sqrt{a_{j k}^{2}+b_{j k}^{2}}, \\
& \text { i.e. } \sqrt{\left(\lambda-a_{j j}\right)^{2}+\left(\mu-b_{j j}\right)^{2}} \leq \sum_{k \neq j} \sqrt{a_{j k}^{2}+b_{j k}^{2}} .
\end{aligned}
$$

Now, $\left|\lambda-a_{j j}\right| \leq \sqrt{\left(\lambda-a_{j j}\right)^{2}+\left(\mu-b_{j j}\right)^{2}} \leq \sum_{k \neq j} \sqrt{a_{j k}^{2}+b_{j k}^{2}}$,

i.e. $-\sum_{k \neq j} \sqrt{a_{j k}^{2}+b_{j k}^{2}} \leq \lambda-a_{j j} \leq \sum_{k \neq j} \sqrt{a_{j k}^{2}+b_{j k}^{2}}$,

i.e. $a_{j j}-\sum_{k \neq j} \sqrt{a_{j k}^{2}+b_{j k}^{2}} \leq \lambda \leq a_{j j}+\sum_{k \neq j} \sqrt{a_{j k}^{2}+b_{j k}^{2}}$.

Therefore, making use of the fact that $a_{j k} \in\left[\underline{a_{j k}}, \overline{a_{j k}}\right]$, we get

$\underline{a_{j j}}-\sum_{k \neq j} \sqrt{\left|\left[\underline{a_{j k}}, \overline{a_{j k}}\right]\right|^{2}+\left|\left[\underline{b_{j k}}, \overline{b_{j k}}\right]\right|^{2}} \leq \lambda \leq \overline{a_{j j}}+\sum_{k \neq j} \sqrt{\left|\left[\underline{a_{j k}}, \overline{a_{j k}}\right]\right|^{2}+\left|\left[\underline{b_{j k}}, \overline{b_{j k}}\right]\right|^{2}}$,

for $j \in\{1, \ldots, n\}$.

Similarly for the imaginary part, we have

$\underline{b_{j j}}-\sum_{k \neq j} \sqrt{\left|\left[\underline{a_{j k}}, \overline{a_{j k}}\right]\right|^{2}+\left|\left[\underline{b_{j k}}, \overline{b_{j k}}\right]\right|^{2}} \leq \mu \leq \overline{b_{j j}}+\sum_{k \neq j} \sqrt{\left|\left[\underline{a_{j k}}, \overline{a_{j k}}\right]\right|^{2}+\left|\left[\underline{b_{j k}}, \overline{b_{j k}}\right]\right|^{2}}$,

for $j \in\{1, \ldots, n\}$. 
Theorem 10. Let $\boldsymbol{A}+i \boldsymbol{B}$ be a complex interval matrix, where $\boldsymbol{A}=[\underline{\boldsymbol{A}}, \bar{A}]$ and $\boldsymbol{B}=[\underline{B}, \bar{B}]$ are two real interval matrices. Then for every eigenvalue $\lambda+i \mu$ of $\boldsymbol{A}+i \boldsymbol{B}$, we have

$$
\begin{aligned}
& \underline{a_{k k}}-\sum_{j \neq k} \sqrt{\mid\left[\left.\underline{\left.a_{j k}, \overline{a_{j k}}\right]}\right|^{2}+\left|\left[\underline{b_{j k}}, \overline{b_{j k}}\right]\right|^{2}\right.} \leq \lambda \leq \overline{a_{k k}}+\sum_{j \neq k} \sqrt{\left|\left[\underline{a_{j k}}, \overline{a_{j k}}\right]\right|^{2}+\left|\left[\underline{b_{j k}}, \overline{b_{j k}}\right]\right|^{2}}, \\
& \underline{b_{k k}}-\sum_{j \neq k} \sqrt{\mid\left[\left.\underline{\left.a_{j k}, \overline{a_{j k}}\right]}\right|^{2}+\mid\left[\left.\underline{\left.b_{j k}, \overline{b_{j k}}\right]}\right|^{2}\right.\right.} \leq \mu \leq \overline{b_{k k}}+\sum_{j \neq k} \sqrt{\mid\left[\left.\underline{\left.a_{j k}, \overline{a_{j k}}\right]}\right|^{2}+\left|\left[\underline{b_{j k}} \overline{b_{j k}}\right]\right|^{2}\right.},
\end{aligned}
$$

for $k \in\{1, \ldots, n\}$.

Proof. Making use of the phenomenon that the eigenvalues of $\boldsymbol{A}$ and $\boldsymbol{A}^{T}$ are the same, for $k \in\{1, \ldots, n\}$ and $A+i B \in \boldsymbol{A}+i \boldsymbol{B}$ from Theorem 8, we have

$$
\begin{aligned}
& \left|(\lambda+i \mu)-\left(a_{k k}+i b_{k k}\right)\right| \leq \sum_{j \neq k} \sqrt{a_{j k}^{2}+b_{j k}^{2}}, \\
& \text { i.e. } \sqrt{\left(\lambda-a_{k k}\right)^{2}+\left(\mu-b_{k k}\right)^{2}} \leq \sum_{j \neq k} \sqrt{a_{j k}^{2}+b_{j k}^{2}} . \\
& \text { Now, }\left|\lambda-a_{k k}\right| \leq \sqrt{\left(\lambda-a_{k k}\right)^{2}+\left(\mu-b_{k k}\right)^{2}} \leq \sum_{j \neq k} \sqrt{a_{j k}^{2}+b_{j k}^{2}}, \\
& \text { i.e. }-\sum_{j \neq k} \sqrt{a_{j k}^{2}+b_{j k}^{2}} \leq \lambda-a_{k k} \leq \sum_{j \neq k} \sqrt{a_{j k}^{2}+b_{j k}^{2}}, \\
& \text { i.e. } a_{k k}-\sum_{j \neq k} \sqrt{a_{j k}^{2}+b_{j k}^{2}} \leq \lambda \leq a_{k k}+\sum_{j \neq k} \sqrt{a_{j k}^{2}+b_{j k}^{2}} .
\end{aligned}
$$

Therefore, making use of the fact that $a_{j k} \in\left[a_{j k}, \overline{a_{j k}}\right]$, we get $\underline{a_{k k}}-\sum_{j \neq k} \sqrt{\left|\left[\underline{a_{j k}}, \overline{a_{j k}}\right]\right|^{2}+\left|\left[\underline{b_{j k}}, \overline{b_{j k}}\right]\right|^{2}} \leq \lambda \leq \overline{a_{k k}}+\sum_{j \neq k} \sqrt{\left|\left[\underline{a_{j k}}, \overline{a_{j k}}\right]\right|^{2}+\left|\left[\underline{b_{j k}}, \overline{b_{j k}}\right]\right|^{2}}$, for $k \in\{1, \ldots, n\}$.

Similarly, for the imaginary part we have,

$\underline{b_{k k}}-\sum_{j \neq k} \sqrt{\left|\left[\underline{a_{j k}}, \overline{a_{j k}}\right]\right|^{2}+\left|\left[\underline{b_{j k}}, \overline{b_{j k}}\right]\right|^{2}} \leq \mu \leq \overline{b_{k k}}+\sum_{j \neq k} \sqrt{\left|\left[\underline{a_{j k}}, \overline{a_{j k}}\right]\right|^{2}+\mid\left[\underline{\left.b_{j k}, \overline{b_{j k}}\right]\left.\right|^{2}}\right.}$, for $k \in\{1, \ldots, n\}$.

Corollary 1. The common bound produced by Theorem 9 and Theorem 10 would be the optimal bounds of the eigenvalues for a complex interval matrix.

\section{EXAMPLES}

In the following examples the interval eigenvalue enclosures are given by Rohn (Theorem 5) and Hladík (Theorem 7), and compared with the proposed Theorem 9. In Figs 1-5 we plot approximation of the set of the eigenvalues by using Monte Carlo (MC) simulation, as well as different enclosures of eigenvalues.

Example 1. Let us consider the interval matrix

$$
\boldsymbol{A}=\left(\begin{array}{cc}
{[1,2]} & {[0.01,0.1]} \\
{[0.001,0.01]} & {[6,8]}
\end{array}\right) .
$$

By the Rohn theorem [9] we have $\lambda \in[0.4986,8.0014]$ and $\mu \in[-1.0260,1.0260]$.

Using the present theorems, we have $\lambda \in[0.9,2.1] \cup[5.99,8.01]$ and $\mu \in[-0.01,0.01]$.

Hladik et. al. [8] produced $\lambda \in[0.4904,2.5094] \cup[5.9906,8.0096]$.

Hladik [15] and Rohn [7] produced the same bound as the Rohn formulae [9]. Combining all, we have $\lambda \in[0.9,2.1] \cup[5.99,8.0014]$ and $\mu \in[-0.01,0.01]$. The bounds are illustrated in Fig. 1 . 


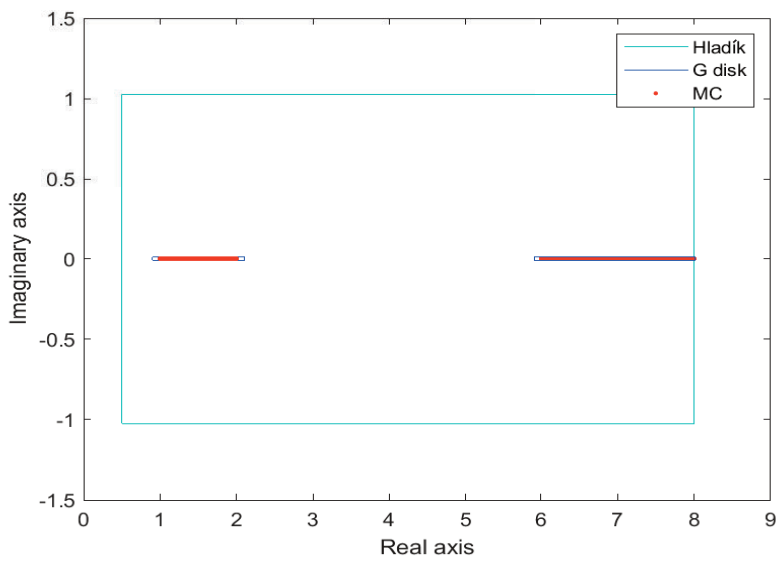

Fig. 1. Eigenvalue set and enclosures of Example 1.

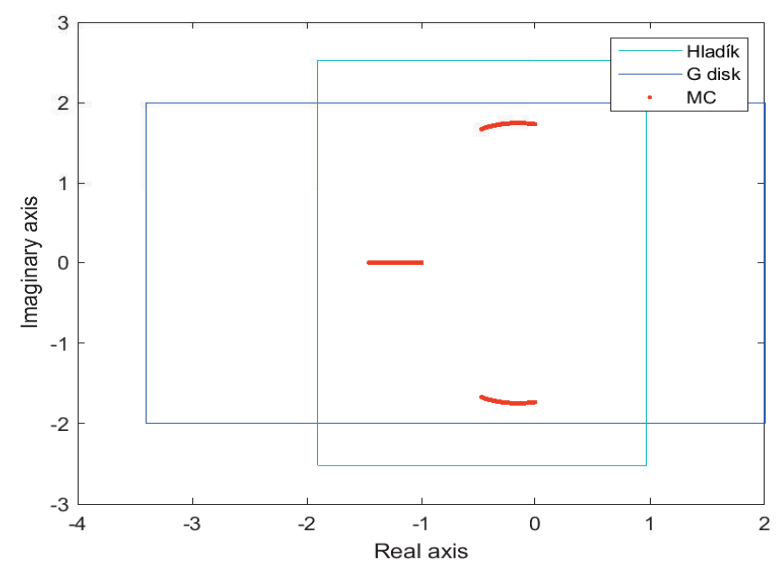

Fig. 2. Eigenvalue set and enclosures of Example 2.

Example 2. Consider the interval matrix [15, Example 2]

$$
\boldsymbol{A}=\left(\begin{array}{ccc}
0 & -1 & -1 \\
2 & {[-1.399,-0.001]} & 0 \\
1 & 0.5 & -1
\end{array}\right)
$$

By the Rohn bounds [9] we have $\lambda \in[-1.9068,0.9702]$ and $\mu \in[-2.5191,2.5191]$.

Using the proposed theorems, we have $\lambda \in[-3.399,2]$ and $\mu \in[-2,2]$.

Although the real bound is not good, it produces a better bound for the imaginary part. Moreover, the imaginary bound is better than the one produced by the exponential method by Hladik [15] and Hertz [1]. The bounds are given in Fig. 2.

Example 3. Consider the complex interval matrix $\boldsymbol{A}+i \boldsymbol{B}$, where

$$
\boldsymbol{A}=\left(\begin{array}{cc}
{[1,2]} & {[0.5,0.7]} \\
{[-0.9,-0.7]} & {[5,8]}
\end{array}\right) \text { and } \boldsymbol{B}=\left(\begin{array}{cc}
{[3,4]} & {[0.3,0.4]} \\
{[0.8,0.9]} & {[6,8]}
\end{array}\right)
$$

By the Hertz theorem [14] we have $\lambda \in[-1.2669,9.2669]$ and $\mu \in[0.1852,10.3149]$. Hladik [15] and Rohn [7] gave $\lambda \in[-1.0294,9.0294]$ and $\mu \in[0.7571,9.7429]$. 


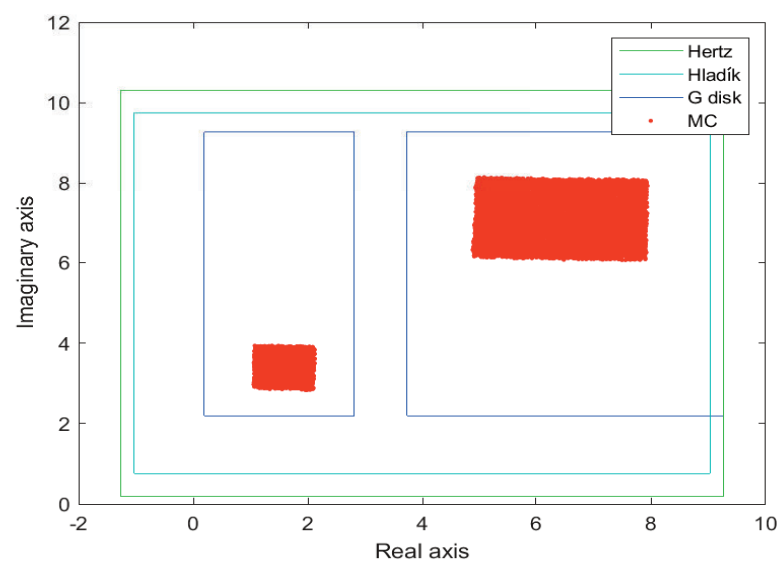

Fig. 3. Eigenvalue set and enclosures of Example 3.

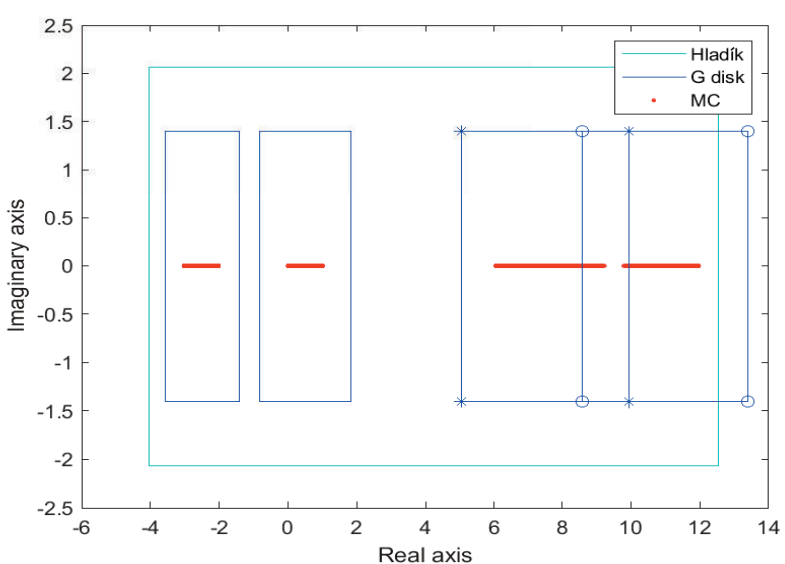

Fig. 4. Eigenvalue set and enclosures of Example 4.

By the present theorems we have $\lambda \in[0.1938,2.8062] \cup[3.7272,9.2728]$ and $\mu \in[2.1938,4.8062] \cup[4.7272$, 9.2728].

The Hertz theorem cannot determine gaps between the interval eigenvalues but the present theorem can. The bounds are depicted in Fig. 3.

Example 4. Let us consider the interval matrix

$$
\boldsymbol{A}=\left(\begin{array}{cccc}
{[0,1]} & {[0.1,0.2]} & {[0.11,0.15]} & {[-0.3,-0.2]} \\
{[0.13,0.16]} & {[-3,-2]} & {[0.4,0.5]} & {[-0.4,-0.2]} \\
{[0.14,0.17]} & {[0.22,0.28]} & {[6,9]} & {[-0.7,-0.5]} \\
{[0.3,0.5]} & {[-0.1,-0.01]} & {[0.2,0.3]} & {[10,12]}
\end{array}\right)
$$

By Rohn [9] we have $\lambda \in[-4.0357,12.5264]$ and $\mu \in[-2.0689,2.0689]$. Hladik [15] and Rohn [7] produced the same bound as the Rohn formulae [9].

Using the proposed theorems, we have $\lambda \in[-3.58,-1.42] \cup[-0.83,1.83] \cup[5.05,9.95] \cup[8.6,13.4]$ and $\mu \in[-1.4,1.4]$. The bounds are shown in Fig. 4. 


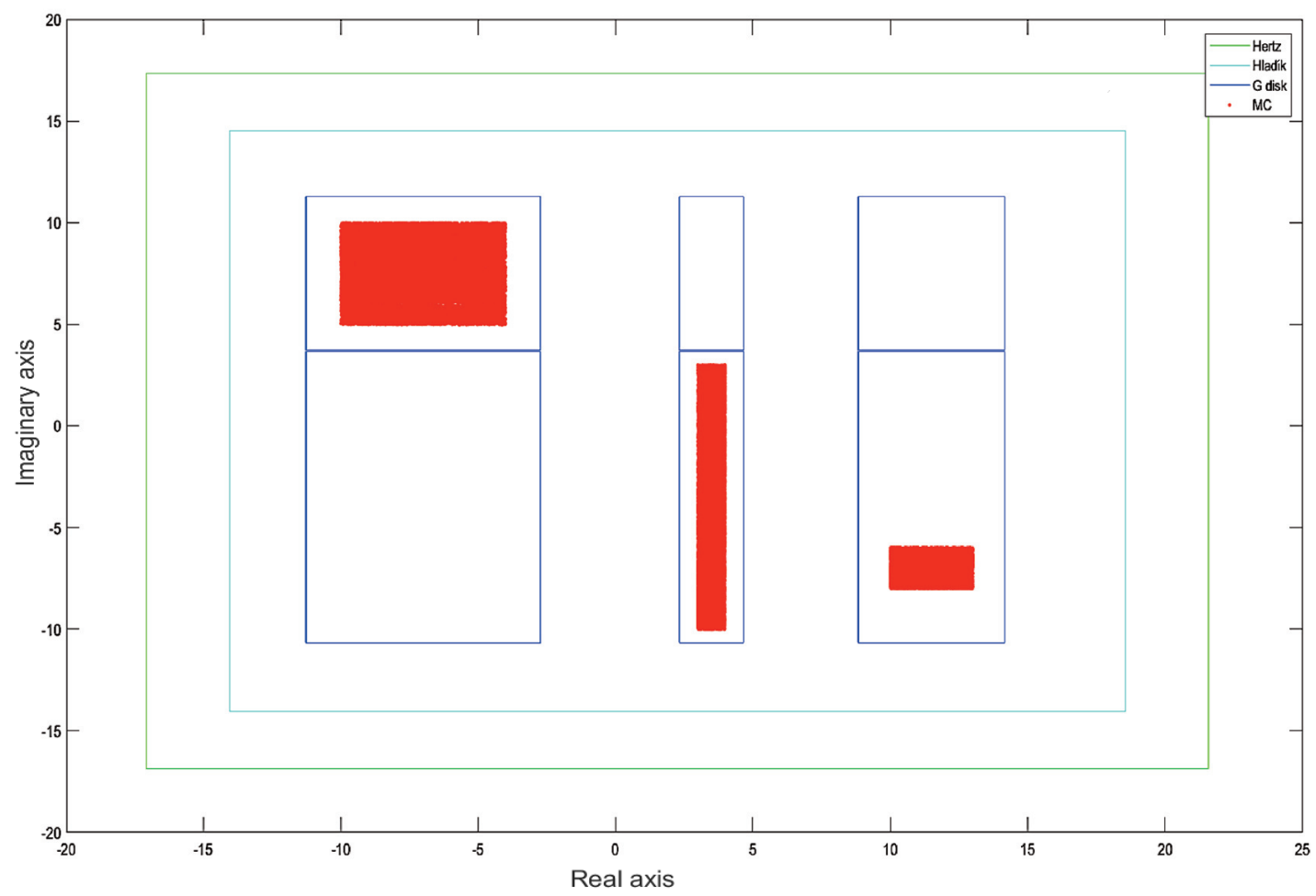

Fig. 5. Eigenvalue set and enclosures of Example 5.

Example 5. Consider the 3-by-3 complex interval matrix $\boldsymbol{A}+\boldsymbol{i B}$, where

$$
\boldsymbol{A}=\left(\begin{array}{ccc}
{[3,4]} & {[0.2,0.3]} & {[-0.2,-0.1]} \\
{[0.11,0.19]} & {[-10,-4]} & {[-0.5,0.4]} \\
{[0.3,0.4]} & {[-0.1,0.4]} & {[10,13]}
\end{array}\right) \text { and } \boldsymbol{B}=\left(\begin{array}{ccc}
{[-10,3]} & {[-0.1,0.2]} & {[0.19,0.23]} \\
{[0.3,0.6]} & {[5,10]} & {[-0.4,-0.2]} \\
{[-0.39,-0.21]} & {[0.4,0.45]} & {[-8,-6]}
\end{array}\right)
$$

By Hertz [14] we have $\lambda \in[-17.0752,21.5727]$ and $\mu \in[-16.8598,17.3649]$.

Hladik [15] and Rohn [7] gave $\lambda \in[-14.0455,18.5467]$ and $\mu \in[-14.0497,14.5374]$.

By the present theorems we have $\lambda \in[-11.2697,-2.7303] \cup[2.3346,4.6654] \cup[8.8393,14.1607]$ and $\mu \in$ $[-10.6654,3.6654] \cup[-9.1607,-4.8393] \cup[3.7303,11.2697]$. The bounds are pictured in Fig. 5.

It can be seen from the previous examples that Theorem 9 is giving better results than Rohn and Hladík. The applications of this phenomenon are discussed in the next section.

\section{DISCUSSION}

If we observe the behavioural changes of the Gershgorin disk theorem on complex interval matrices for enclosing complex interval eigenvalues, we can see that for diagonal interval matrices Rohn [9], Hertz [14], and the Gershgorin disk theorem have provided the same interval eigenvalue bounds. Now, if we start making the non-principal diagonal entries from zero intervals to nonzero intervals, then Theorem 9 will enclose interval eigenvalues better than Rohn [9] and Hertz [14]. Furthermore, if we increase more the width of non-principal diagonal entries, then Rohn [9] and Hertz [14] will become beneficial. 


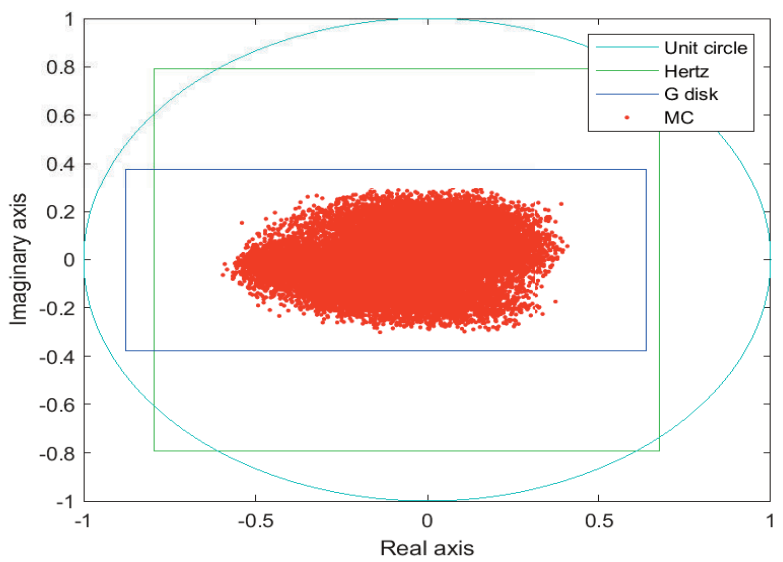

Fig. 6. Schur stability.

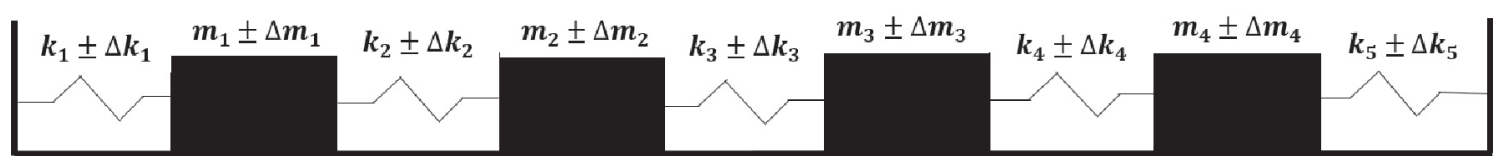

Fig. 7. Spring-mass system.

\subsection{Applications}

Schur stability: A matrix $A+i B$ is called Schur stable if all the eigenvalues lie in the open unit disk centred at the origin. Now, consider the linear time-invariant dynamical system with uncertainty $x^{\prime}(t)=(A+i B) x(t)$, where $A+i B \in \boldsymbol{A}+i \boldsymbol{B}$ with

$$
\boldsymbol{A}=\left(\begin{array}{cc}
{[-0.5,0.26]} & {[0.17,0.23]} \\
{[-0.21,0.16]} & {[-0.2,0.2]}
\end{array}\right) \text { and } \boldsymbol{B}=\left(\begin{array}{cc}
{[0,0]} & {[-0.1,0.3]} \\
{[-0.1,0.2]} & {[0,0]}
\end{array}\right)
$$

The Hertz formulae [14] gave $\lambda \in[-0.7963,0.6763]$ and $\mu \in[-0.7928,0.7928]$. From this bound one cannot conclude that the matrix $\boldsymbol{A}+i \boldsymbol{B}$ is Schur stable. On the other hand, Theorem 9 produces bounds as $\lambda \in[-0.8780,0.6380]$ and $\mu \in[-0.3780,0.3780]$, i.e. each eigenvalue lies in the open unit disk centred at the origin, concluding that $\boldsymbol{A}+i \boldsymbol{B}$ is Schur stable. The pictorial depiction is provided in Fig. 6 .

Spring-mass system: We know that the characteristics of spring-mass systems (Fig. 7) can be determined by the eigenvalues of mass and stiffness matrices of that system.

For an uncertain spring-mass system we consider the mass matrix as $M_{c}=\operatorname{diag}(1,1,1,1)$ with mass uncertainty $M_{\Delta}=\operatorname{diag}(0,0,0,0)$ and stiffness matrix

$$
K_{c}=\left(\begin{array}{cccc}
3000 & -0.1 & 0 & 0 \\
-0.1 & 5000 & -0.15 & 0 \\
0 & -0.15 & 7000 & -0.3 \\
0 & 0 & -0.3 & 9000
\end{array}\right)
$$

with stiffness uncertainty

$$
K_{\Delta}=\left(\begin{array}{cccc}
25 & 0.2 & 0 & 0 \\
0.2 & 35 & 0.17 & 0 \\
0 & 0.17 & 45 & 0.1 \\
0 & 0 & 0.1 & 55
\end{array}\right)
$$

corresponding to a spring-mass system with four degrees of freedom in Fig. 7. 
Now, by computing the interval eigenvalue bounds by different methods and according to the present method, we can see the tightness of our result. Consequently, we are able to get a tighter solution for the uncertain spring-mass system.

By the Rohn formulae [9] we have $\lambda \in[2944.9989,3.055 .0010] \cup[4944.9989,5055.0010] \cup[6944.9989$, 7.055.0010] $\cup[8944.9990,9055.0011]$.

By Leng and He [4] one has $\lambda \in[2944.8999,3055.0100] \cup[4944.8999,5055.0100] \cup[6944.8999$, 7.055.0100] $\cup[8944.9000,9055.1001]$.

By the present formulae we have $\lambda \in[2974.7,3025.3] \cup[4964.5613,5035.4387] \cup[6954.4877,7045.5123]$

$\cup[8944.6,9055.4]$.

\section{CONCLUSIONS}

The enclosures of the eigenvalue set of complex interval matrices given by the present Gershgorin disk theorem may be considered as initial bounds. Then different algorithms [12,17] can be used for further better approximations of the eigenvalue set.

Although direct application of the above approach is not beneficial for the interval matrices whose nonprinciple diagonal entries have a large width, one can investigate the behaviours of direct application of the Gershgorin disk theorem on interval matrices for bounding eigenvalues through translations.

\section{ACNOWLEDGEMENTS}

Suman Maiti is grateful to the Council of Scientific and Industrial Research (CSIR), India, for the financial support to perform this work. The publication costs of this article were covered by the Estonian Academy of Sciences.

\section{REFERENCES}

1. Hertz, D. The extreme eigenvalues and stability of real symmetric interval matrices. IEEE Trans. Autom. Control, 1992, 37(4), 532-535.

2. Rohn, J. Stability of interval matrices: the real eigenvalue case. IEEE Trans. Autom. Control, 1992, 37(10), 1604-1605.

3. Ahn, H.-S., Chen, Y. Q. and Podlubny, I. Robust stability test of a class of linear time-invariant interval fractional-order system using Lyapunov inequality. Appl. Math. Comput., 2007, 187(1), 27-34.

4. Leng, H. and He, Z. Computing eigenvalue bounds of structures with uncertain-but-non-random parameters by a method based on perturbation theory. Commun. Numer. Methods Eng., 2007, 23(11), 973-982. https://onlinelibrary.wiley.com/doi/abs/10.1002/ cnm.936

5. Leng, H., He, Z. and Yuan, Q. Computing bounds to real eigenvalues of real-interval matrices. Int. J. Numer. Methods Eng., 2008, 74(4), 523-530. https://onlinelibrary.wiley.com/doi/abs/10.1002/nme.2179

6. Deif, A. The interval eigenvalue problem. ZAMM (J. Appl. Math. Mech.), 1991, 71(1), 61-64. https://onlinelibrary.wiley.com/doi/ abs/10.1002/zamm.19910710117

7. Rohn, J. A Handbook of Results on Interval Linear Problems. Czech Academy of Sciences, Prague, 2005.

8. Hladík, M., Daney, D. and Tsigaridas, E. Bounds on real eigenvalues and singular values of interval matrices. SIAM J. Matrix Anal. Appl., 2010, 31(4), 2116-2129. https://doi.org/10.1137/090753991

9. Rohn, J. Bounds on eigenvalues of interval matrices. ZAMM (J. Appl. Math. Mech.), 1998, 78(S3), 1049-1050. https://onlinelibrary.wiley.com/doi/abs/10.1002/zamm.19980781593

10. Bauer, F. L. and Fike, C. T. Norms and exclusion theorems. Numer. Math., 1960, 2, 137-141. https://doi.org/10.1007/BF01386217

11. Chu, K.-w. E. Generalization of the Bauer-Fike theorem. Numer. Math., 1986, 49, 685-691. https://doi.org/10.1007/BF01389736

12. Hladík, M., Daney, D. and Tsigaridas, E. A filtering method for the interval eigenvalue problem. Appl. Math. Comput., 2011, 217(12), 5236-5242. http://www.sciencedirect.com/science/article/pii/S0096300310010702

13. Mayer, G. A unified approach to enclosure methods for eigenpairs. ZAMM (J. Appl. Math. Mech.), 1994, 74(2), 115-128.

14. Hertz, D. Interval analysis: eigenvalue bounds of interval matrices. In Encyclopedia of Optimization (Floudas, C. and Pardalos, P., eds). Springer, Boston, MA, 2008. https://doi.org/10.1007/978-0-387-74759-0_295

15. Hladík, M. Bounds on eigenvalues of real and complex interval matrices. Appl. Math. Comput., 2013, 219(10), 5584-5591. 
16. Matcovschi, M.-H. and Pastravanu, O. Novel estimations for the eigenvalue bounds of complex interval matrices. Appl. Math. Comput., 2014, 234, 645-666.

17. Roy, F. and Gupta, D. K. Sufficient regularity conditions for complex interval matrices and approximations of eigenvalues sets. Appl. Math. Comput., 2018, 317, 193-209.

18. Chakraverty, S., Sahoo, D. M. and Mahato, N. R. Concepts of Soft Computing: Fuzzy and ANN with Programming. Springer, Singapore, 2019.

19. Horn, R. A. and Johnson, C. R. Matrix Analysis. Cambridge University Press, Cambridge, 1985.

20. Meyer, C. Matrix Analysis and Applied Linear Algebra. Society for Industrial and Applied Mathematics, 2000. https://books.google.co.in/books?id=Brpp1Cvzs14C

21. Gershgorin, S. Über die Abgrenzung der Eigenwerte einer Matrix. Izv. Akad. Nauk SSSR, 1931, 7, 749-754.

\section{Gershgorini ringiteoreem komplekssete intervallmaatriksite käsitlemisel}

\section{Suman Maiti ja Snehashish Chakraverty}

Artiklis pakutakse välja kasutada komplekssete maatriksite omaväärtuste intervallide leidmiseks Gershgorini ringiteoreemi. Tegemist on mitteiteratiivse meetodiga, mis võimaldab leida omaväärtuste reaal- ja imaginaarosade alam- ja ülemrajasid. Peale selle käsitletakse omaväärtuste inervallide klastrite vaheliste kauguste määramist. Saadud tulemusi võrreldakse eelnevatega, mis on saadud komplekssete maatriksite omaväärtuste intervallide leidmisel. Väljatöötatud meetodid omavad rakendusi dünaamiliste süsteemide stabiilsuse uurimisel. 